\title{
On the Solution to Pre-Buckling of a Thin Rectangular Plate Subjected to a Thick Strip In-Plane Loading
}

\author{
JACOB NAGLER \\ NIRC \\ Haifa, Givat Downes \\ ISRAEL
}

\begin{abstract}
The current paper deals with the problem of the simply supported thin rectangular plate subjected to the intermediate strip in-plane loading. Based on the strain energy method (Fourier ansatz), the critical (minimum value) of buckling stress occurrence was determined in a general form dependent only on the strip thickness, strip location, plate width and stress magnitude. Compatible with the classical columns Euler method it was found that the plate stability is decreased with the increasing of the plate width due to larger induced stresses. Also, strip location relative to the support region was found to influence the buckling (same analogy to the Euler buckling theory; consider the strip as a both sides pressed rod). Additionally, the strip width parameter increase is likely to cause larger buckling stress. Moreover, expressions that includes both axial and transverse loads for different extended cases configurations were also derived and examined based on the strain energy method alongside explanation for possible applications (thin aluminum plate welding). In a general view, it was found that the cases of combined axial and perpendicular loading action are less stabilized than cases where only one kind of loading configuration is participated. Finally, the buckling stress was found to agree qualitatively with the cited literature.
\end{abstract}

Key-Words: - static, buckling; in-plane loading; buckling stress; rectangular plate; strip.

Received: May 10, 2021. Revised: October 2, 2021. Accepted: October 16, 2021. Published: October 27, 2021.

\section{Introduction}

Rectangular plate subjected to an in-plane stress in the geometry shape of rectangular strip has a lot of importance in the ship industry (side plates [1] and stiffened ribs plates [2]), and structural engineering (stiffened plates [3], perforated thin plates [4]), manufacturing (cold rolling process [5-6]) and composite plates (ply and film plates [7-9] FGM plates [10-11] and other types of plate involving the viscoelastic form [12-13]). The prevailing common classical solution of compressed stress due to rib influence can be found in classical books of solid \& structural mechanics [14-17].

The loading configurations at the plate boundaries can be divided into static and dynamic loads [19]. In addition, post- or pre- buckling state [19] is another way of classification and final method to distinguish is the applying loads type and way of operation [13, 20-24].

The calculations methods to cope with the problem diverse from Rayleigh - Ritz methods [10], numerical methods [22], exact analytic solutions [25] and semi-analytic solution [26-27].
Specifically, the case of intermediate load in the pre-buckling state in the in-plane was investigated by [25-29] which based on the direct thin plate theory displacement equation. However, the current study extent the studies of Xiang et al. [25], Yao et al., [26], Mijušković et al. [27] and Wang et al. [2829] by developing a general analytic solution to the pre -buckling state of the simply supported rectangular thin plate subjected to a thick strip that is generally located in the plate plane based on the strain energy theory. The obtained expression will be examined and compared qualitatively to the relevant literature references [2, 14, 16, 18, 25].

Before beginning with the analytic model, it will be mentioned that there are several main plate theoretical model in the literature for different regimes, like: Kirchhoff-Love [31-33] (simple displacement relations, all kinds of thinner plate thickness), Ulfyand-Mindlin [34-35] (plate thickness to planar dimensions ratio is of the order of one tenth), Mindlin-Reissner [36-38] (the bending stress is assumed to behave linearly while the shear stress is supposed to be quadratic through the plate thickness), Reissner-Stein [39] (improved Saint-Venant theory for cantilever plates) and von Karman [40-44] (general and most veteran model 
from 1910 describing the large displacements of thin flat plate based on nonlinear partial differential equation). Moreover, von Karman model for thin flat elastic plate subjected to stress is an extension of Kirchhoff-Love model and based on differential kinematic equations depicting stress-strain relationships [40-44]. The solution for the obtained non-linear differential equation (large deflection) that is based on Airy's function is not easy and immediate to solve [44]. There are methods that convert the non-linear set of equilibrium [40, 43 44] to energy [40] or guessing appropriate displacement function [41] method which is similar to what we propose here. However, here we concern only partial stress loading region (strip) that act on a supported thin flat elastic plate.

Ulfyand-Mindlin [34 - 35] and MindlinReissner [36, 38] plates have their own complicated assumptions (as noted above) with appropriated differential equations relationships between stresses and strains. Yet, Brunelle and Robertson [34] (deals with different boundary conditions and full stress loading on the opposite edges) and Bui et al. [37] (only generalized scheme without solving or specifying a particular case) have formulated it by using energy form which is similar to the current study.

Another dominant model-case which is similar to the current study energy formulation has been produced by Reissner-Stein [39] for cantilever elastic thing plate under torsional and transverse moments bending which is different from our model (boundary conditions).

As though, particularly, the current model is based on total potential energy function minimization formulation that appear (for example) Reissner-Stein [39] and also known to be called Fourier Ansatz model which is derived from the linear theory (i.e. governing energy is functional quadratic). Concentrating on thin, flat elastic plate under partial loading in order to have simple closed analytic solution for future evaluation of applications based on (or involved with) prebuckling stress (heating, punching, etc.).

\section{Problem Formulation}

In a general form, consider a simply supported thin flat elastic plate $(\Delta)$ subjected to thick strip loading with thickness as appear in Fig. 1. Using Cartesian coordinates system as shown in Fig. 1, alongside the general equation of strain energy density for plane stress $\left(\sigma_{z}=0\right)[2,14,18]$ :

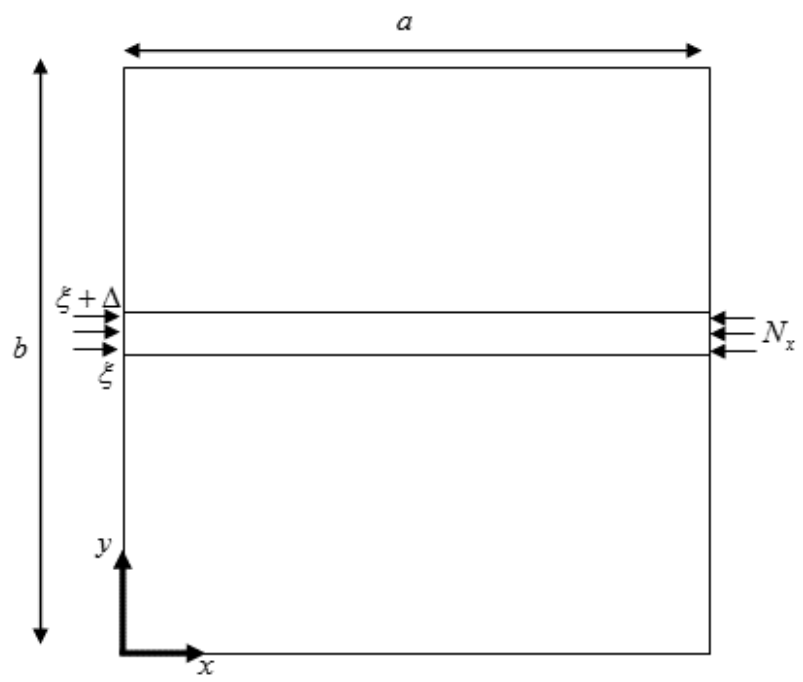

Fig. 1: Simply supported plate subjected to a thick strip loading

$$
\begin{aligned}
& U=\frac{D}{2} \int_{0}^{a} \int_{0}^{b}\left(\frac{\partial^{2} w}{\partial x^{2}}+\frac{\partial^{2} w}{\partial y^{2}}\right)^{2}-2(1-v)\left(\frac{\partial^{2} w}{\partial x^{2}} \frac{\partial^{2} w}{\partial y^{2}}-\frac{\partial^{2} w}{\partial x \partial y}\right)^{2} d y d x= \\
& \frac{D}{2} \int_{0}^{a} \int_{0}^{b}\left[(\Delta w)^{2}-2(1-v)\left(w_{, x x} \cdot w_{, y y}-w_{, x y}\right)^{2}\right] d x d y
\end{aligned}
$$

where $D=\frac{E t^{3}}{12\left(1-v^{2}\right)}, E$ is the Young modulus and $v$ is the Poisson ratio. Additionally, the work expression $(V)$ that is defined only for the strip region $(\xi \leq y \leq \xi+\Delta)$ and controlled by the concentrated normalized (outer) pressures/stresses $N_{x}$ in the strip distributed uniformly over the displacement $w$, will be:

$$
V=\frac{1}{2}\{f\} w^{2}=-\frac{N_{x} t}{2} \int_{0}^{a} \int_{\xi}^{\xi+\Delta}\left(\frac{\partial w}{\partial x}\right)^{2} d y d x
$$

Now, we will assume that the deflection parameter can be represented using the following function:

$$
w(x, y)=\sum_{m=1} \sum_{n=1} A_{m n} \sin \left(\frac{m \pi x}{a}\right) \sin \left(\frac{n \pi y}{b}\right),
$$

which fulfils the following simply supported (S-S) boundary conditions (B.C.): 
One simple support on the one end side:

$w_{1}(x, 0)=w_{1, y y}(x, 0)=0$

One simple support on the one end side:

$w_{1}(x, 0)=w_{1, y y}(x, 0)=0$

One simple support on both end sides:

$w(x, 0)=w_{y y}(x, 0)=0$,

$w(x, b)=w_{y y}(x, b)=0$

First continuous condition: $\left\{\begin{array}{l}w_{1}(x, \xi)=w_{2}(x, \xi) \\ w_{1, y y}(x, \xi)=w_{2, y y}(x, \xi)\end{array}\right.$

Second continuous condition:

$\left\{\begin{array}{l}w_{2}(x, \xi+\Delta)=w_{3}(x, \xi+\Delta) \\ w_{2, y y}(x, \xi+\Delta)=w_{3, y y}(x, \xi+\Delta)\end{array}\right.$

while the constants $A_{n}$ are dependent on the boundary is a scalar function, however, the conditions. Note that $w$ index numbers $1,2,3$ are aided to separate three plate subdomains ( 1 - right side, 2 - strip, 3 - left side).

Hence the stored energy and the virtual work reads, are after substituting (3) into expressions (1) and (2), as:

$U=\frac{D}{2} \int_{0}^{a} \int_{0}^{b}\left(\frac{\partial^{2} w}{\partial x^{2}}+\frac{\partial^{2} w}{\partial y^{2}}\right)^{2}-2(1-v)\left(\frac{\partial^{2} w}{\partial x^{2}} \frac{\partial^{2} w}{\partial y^{2}}-\frac{\partial^{2} w}{\partial x \partial y}\right)^{2} d y d x=$

$\sum_{m} \sum_{n} A_{m n}^{2} \frac{\pi^{4} D a b}{8}\left(\frac{m^{4}}{a^{4}}+\frac{n^{4}}{b^{4}}+2 v\left(\frac{m n}{a b}\right)^{2}-\frac{9}{8} \pi^{4} A_{m n}^{2}(1-v)\left(\frac{m n}{a b}\right)^{4}\right)$

In order to simplify, we will assume $v=1$ and consider only the bending influence, such as Eq. (4) will become:

$U=\frac{D}{2} \int_{0}^{a} \int_{0}^{b}\left(\frac{\partial^{2} w}{\partial x^{2}}+\frac{\partial^{2} w}{\partial y^{2}}\right)^{2} d y d x=\sum_{n m} A_{m n}^{2} \frac{D a b \pi^{4}}{8}\left(\frac{m^{2}}{a^{2}}+\frac{n^{2}}{b^{2}}\right)^{2}$

Note that $m$ and $n$ indicate the numbers of halfwaves in each direction.

$$
\begin{aligned}
& V=\frac{1}{2}\{f\} w_{, x}^{2}=-\frac{N_{x} t}{2} \int_{0}^{a} \int_{\xi}^{\xi+\Delta}\left(\frac{\partial w}{\partial x}\right)^{2} d y d x= \\
& -\frac{N_{x} t}{2}\left(\frac{m \pi}{a}\right)^{2} \int_{0}^{a} \sum_{m} \cos ^{2}\left(\frac{m \pi x}{a}\right) d x \cdot \int_{\xi}^{\xi+\Delta}\left\{\sum_{n} A_{m n} \sin \left(\frac{n \pi y}{b}\right)\right\}^{2} d y= \\
& -\frac{N_{x} t m^{2} \pi^{2}}{8 a} \cdot \sum_{n m} A_{m n}^{2}\left\{\Delta-\frac{b}{2 n \pi}\left[\begin{array}{l}
\sin \left(\frac{2 n \pi(\xi+\Delta)}{b}\right) \\
-\sin \left(\frac{2 n \pi \xi}{b}\right)
\end{array}\right]\right\}
\end{aligned}
$$

since $\int_{0}^{a} \sum_{m} \cos ^{2}\left(\frac{m \pi x}{a}\right) d x=a / 2$

Hence, the overall potential energy of the system will supply:

$$
\begin{aligned}
& \Pi=U+V= \\
& \int_{0}^{a} \int_{0}^{b}\left[\begin{array}{l}
\left.\frac{D}{2}\left(\nabla^{2} w\right)^{2}-2(1-v)\left(w_{, x x} \cdot w_{, y y}-w_{, x y}\right)^{2}\right] d x d y= \\
-\frac{1}{2}\{f\} w_{, x}^{2}
\end{array}\right] \\
& \sum_{n m} A_{m n}^{2} \frac{D a b \pi^{4}}{8}\left(\frac{m^{2}}{a^{2}}+\frac{n^{2}}{b^{2}}\right)^{2} \\
& -\frac{N_{x} t \pi^{2}}{8 a} \cdot \sum_{n m} m^{2} A_{m n}^{2}\left[\Delta-\frac{b}{2 n \pi}\left[\begin{array}{l}
\sin \left(\frac{2 n \pi(\xi+\Delta)}{b}\right) \\
-\sin \left(\frac{2 n \pi \xi}{b}\right)
\end{array}\right]\right\} \geq 0
\end{aligned}
$$

Therefore the limited general minimum expression value for $N_{x}$ will be:

$$
N_{x} \leq \frac{\sum_{n m} A_{m n}^{2} \frac{\pi^{2} D a^{2} b}{t}\left(\frac{m^{2}}{a^{2}}+\frac{n^{2}}{b^{2}}\right)^{2}}{\sum_{n m} m^{2} A_{m n}^{2}\left\{\Delta-\frac{b}{2 n \pi}\left[\begin{array}{l}
\left.\sin \left(\frac{2 n \pi(\xi+\Delta)}{b}\right)\right]-\sin \left(\frac{2 n \pi \xi}{b}\right)
\end{array}\right]\right\}}
$$

The critical value of $N_{x}$ will be (for one $A_{m n}$ term): 


$$
\left.N_{x}\right|_{c r} \leq \frac{\pi^{2} D a^{2} b}{m^{2} t} \cdot \frac{\left(\frac{m^{2}}{a^{2}}+\frac{n^{2}}{b^{2}}\right)^{2}}{\Delta-\frac{b}{2 n \pi}\left[\begin{array}{l}
\left.\sin \left(\frac{2 n \pi(\xi+\Delta)}{b}\right)\right] \\
-\sin \left(\frac{2 n \pi \xi}{b}\right)
\end{array}\right]} .
$$

Alternative method, finding the extreme values of $N_{x}$ by differentiating the inequality $\Pi$ along constant $A_{m n} \neq 0$ as $\frac{\partial \Pi}{\partial A_{m n}}=0$ gives the same value. Now, expression (9) will be approximated for the first minimal critical buckling load with the parameters pair $(m, n)$ to yield:

$$
\left.N_{x}\right|_{c r}=\frac{\pi^{2} D a^{2} b}{m^{2} t} \cdot \frac{\left(\frac{m^{2}}{a^{2}}+\frac{n^{2}}{b^{2}}\right)^{2}}{\Delta+\frac{b}{n \pi}},
$$

such as the sinus expression in the denominator will be bounded

as

$\left|\sin \left(\frac{2 n \pi(\xi+\Delta)}{b}\right)-\sin \left(\frac{2 n \pi \xi}{b}\right)\right| \leq 2$

since

$|\sin (x)|<1$. Note that the solution $\left.N_{x}\right|_{c r}=\frac{\pi^{2} D a^{2} b}{m^{2} t} \cdot \frac{\left(\frac{m^{2}}{a^{2}}+\frac{n^{2}}{b^{2}}\right)^{2}}{\Delta-\frac{b}{n \pi}}$ is not physical (due to the negative denominator, $b>\Delta$ ). Expressions (9)-(10) seem to make sense in the context of $\Delta$ behavior since the critical load $N_{x}$ required to buckle the plate is diminished with the strip width increase. Next step, for $n=1$ (10) has the minimum value and becomes:

$$
\left.N_{x}\right|_{c r}=\frac{\pi^{2} D a^{2} b}{m^{2} t} \cdot \frac{\left(\frac{m^{2}}{a^{2}}+\frac{1}{b^{2}}\right)^{2}}{\Delta+\frac{b}{\pi}}=\frac{\pi^{2} D b}{a^{2} t} \cdot \frac{\left[m+\frac{1}{m}\left(\frac{a}{b}\right)^{2}\right]^{2}}{\Delta+\frac{b}{\pi}} .
$$

Now, using some algebra can lead us to the reduced relation (11), presented as Euler's formula in the form of boundary support:

$$
\left.N_{x}\right|_{c r}=\frac{\pi^{2} D}{b t} \cdot \frac{k}{\Delta \mp \frac{b}{\pi}} ; \quad k=\left(\frac{m b}{a}+\frac{a}{m b}\right)^{2} .
$$

Note that for the case $\Delta=b, \xi=0$ (inserted into Eq. (9)) we obtain the classical solution $[2,14,20]$ :

$$
\left.N_{x}\right|_{c r}=\frac{\pi^{2} D}{b^{2} t} \cdot k ; \quad k=\left(\frac{m b}{a}+\frac{a}{m b}\right)^{2} .
$$

Note that the classical solution (13) is not dependent on the plate width length $b$ whereas expressions (9) and (10) are dependent on $b$ due to the additional strip width $\Delta$.

Finally, the maximum buckling stress for the problem posed by $[25,28-29]$ including two extensions will be presented. Following the previous development, three prominent extent problems shown in Figs. 2 are solved in Table 1 based on the superposition principal. Note that the subdomain 2 definition in case (c) relates to the whole plate without the loads $N_{1}$.
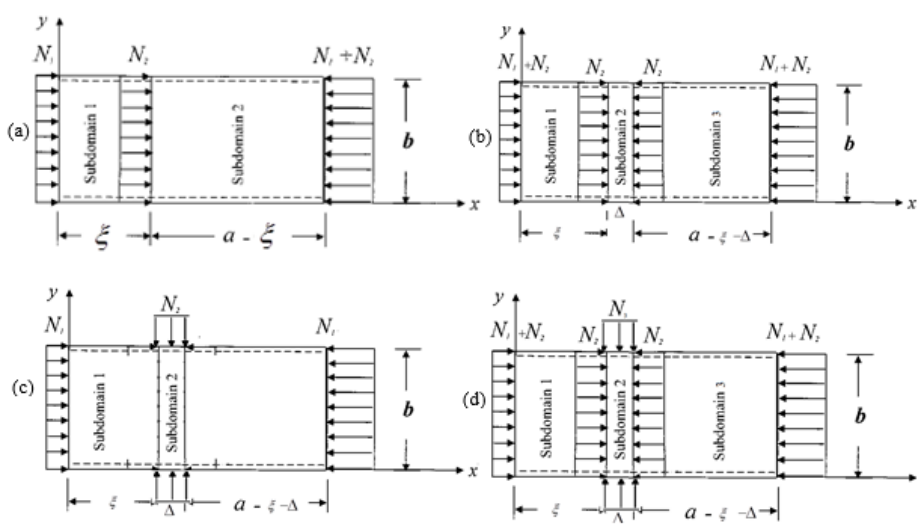

Fig. 2: (a) Simply supported plate subjected to thin intermediate and ended uniaxial loads (taken from [25, 28-29]) (b) Rectangular plate subjected to thick intermediate and ended uniaxial loads (c) Rectangular plate subjected to perpendicular thick load and ended uniaxial loads (d) Rectangular plate subjected to intermediate perpendicular combined with uniaxial thick loads and ended uniaxial loads 
Table 1. Comparison between different load configurations vs. maximum buckling stress

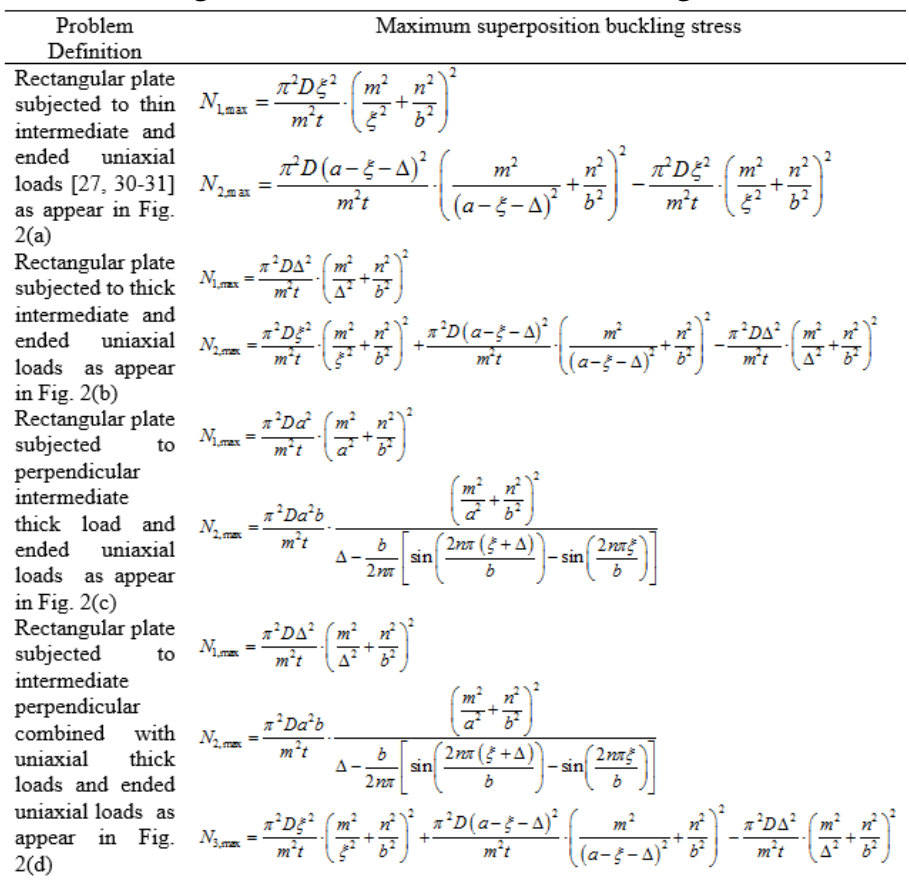

\section{Results and Discussion}

In this section the following parameters will be substituted into Eqs. (9)-(10) and (13) which are represented in Fig 3, as:

$$
\begin{aligned}
& b=1, a=0.5 b, \Delta=0.3 b \\
& 0 \leq a / b \leq 3,0 \leq \Delta / b \leq 3,0 \leq \xi / b \leq 3
\end{aligned}
$$

Respectively, the representative parameters in Figs. 4-7 are as follows:

$$
\begin{aligned}
& a=1, a=0.5 b, \Delta=0.3 b \\
& 0 \leq b / a \leq 3,0 \leq \Delta / a \leq 3,0 \leq \xi / a \leq 3
\end{aligned} .
$$

Examining Fig. 3(a) teaches that all solutions are very close quantitatively and behaves qualitatively the same. Also, the approximate solution (10) seems to be an average solution between the exact solution (9) and the classic (13) solution. The buckle loading seems to decrease until they reach certain critical value of $a / b$ ratio from which they slightly increase but keeps stabilized as soon as the plate is more elongated ( $b$ parameter is reduced or $a$ is increased). The opposite (minimum critical buckling load is decreased) occurs if the plate is flattened (fit with the classical columns Euler method). Note that similar phenomenon occurs in Figs. 4(a)-7(a). Accordingly, it can be assumed approximately that the minimum buckling load is varied linearly according with the geometrical ratio $a / b$ (Fig. 3(a)) and b/a (Figs. 4(a)7(a)), respectively.

Analyzing the strip width $(\Delta)$ influence; one might conclude from observing 3(b)-7(b) that the required critical loading to buckle the plate is being reduced due to the fact that the increase of the strip width is accompanied with larger induced stresses that accelerating the buckling process. Although it was not illustrative presented, the parameters $m, n$ has almost no effect on the buckling load for (relative to the) high strip width length.

Analyzing the strip location $(\xi)$ appearing in Figs. 3(c)-7(c) that expressed in sinus wave of maximum loading in reversed direction at the center of the plate might be explained by the fact that the existing induced stresses are located in further distances from the supports locations. Therefore when the stresses acting in a further distance relative to the supports locations the buckling might be occurred sooner which is also adjust to Euler compressed column theory. Remark that the jump in Figs. 3(c)-7(c) resembles numerically the fact that the location of the loading is the same as its width.

One application that can be used here is when thin aluminium plate is subjected to heating through welding process - by making row of thin welding strips with spaces between them as close as possible to the supports in order to avoid concentrated induced stresses that might cause buckling.

Observing Figs. 4-7 we can see the influence (disturbance) of the transverse perpendicular loading as appear in 6(a) and 7(a). Generally speaking, the cases where both axial and perpendicular loading are acting are less stable than cases where only one kind of loading configuration is participated. Finally, qualitative confirmation with literature $[2,14,16,18,25]$ has been achieved. 
(a)

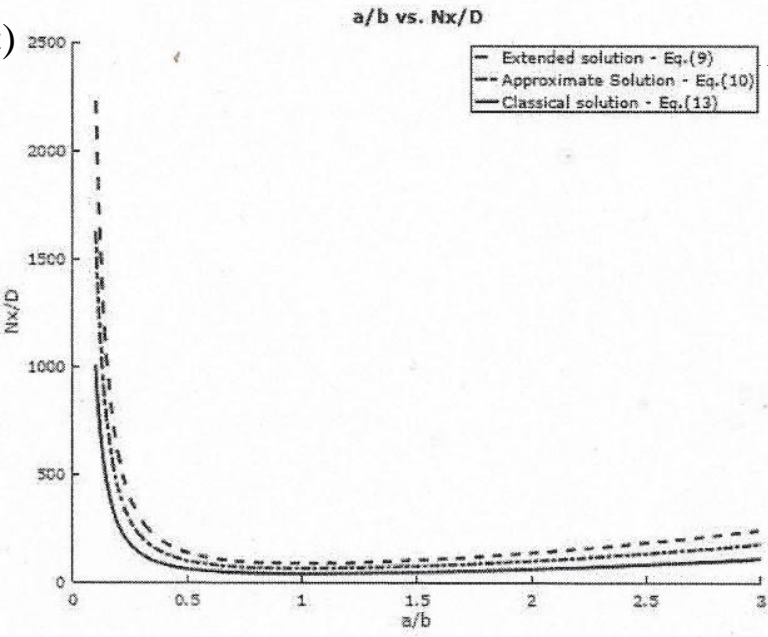

(b)

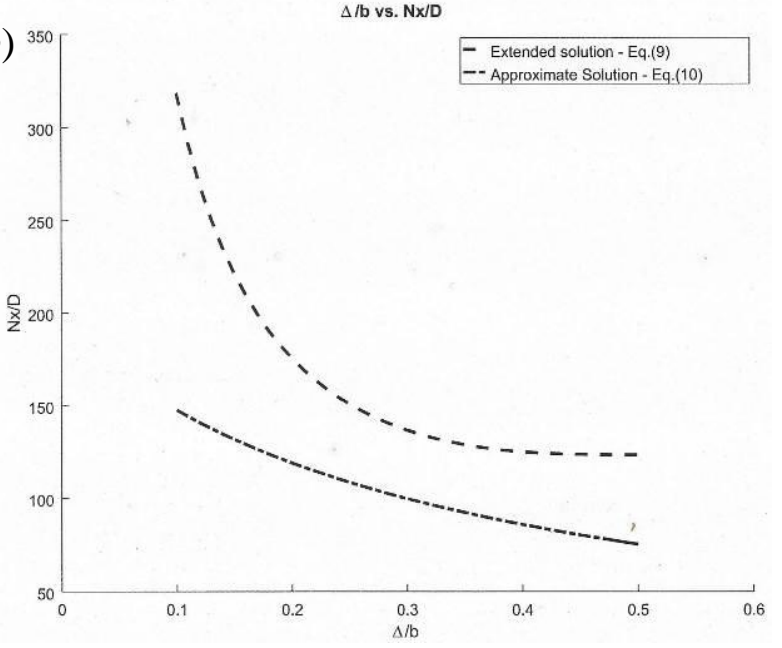

(c)

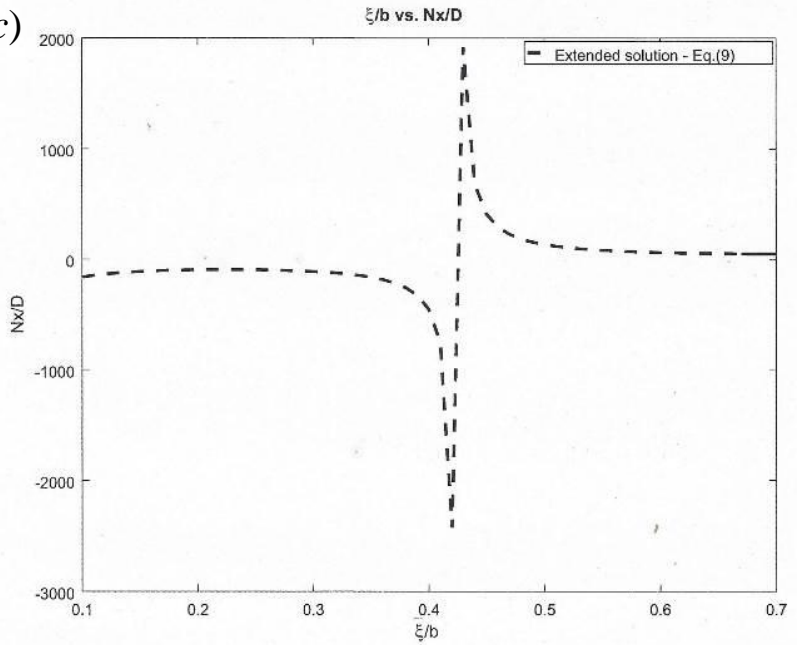

Fig. 3: Stability criteria (critical load) for simply supported plates under intermediate load vs. (a) different geometrical ratio $(a / b)$ values (b) different delta $(\Delta)$ width length values (c) different $\xi$ distance values. (a)

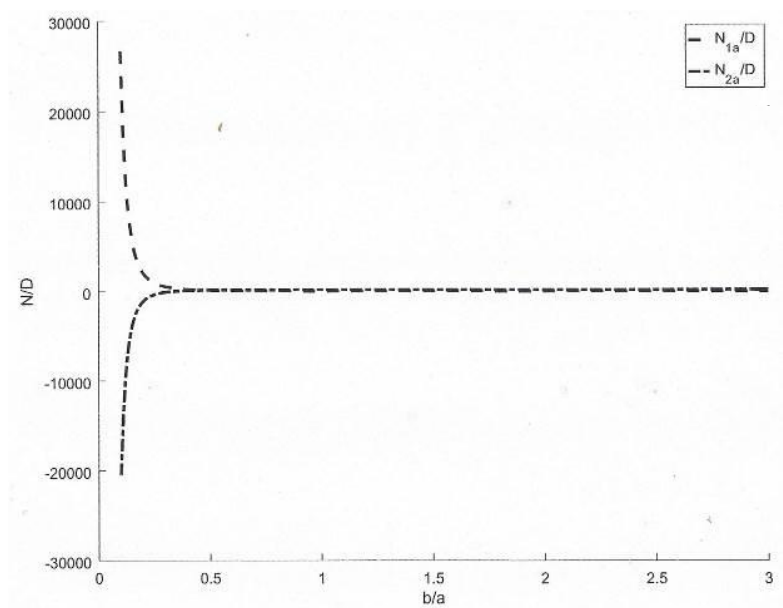

(b)

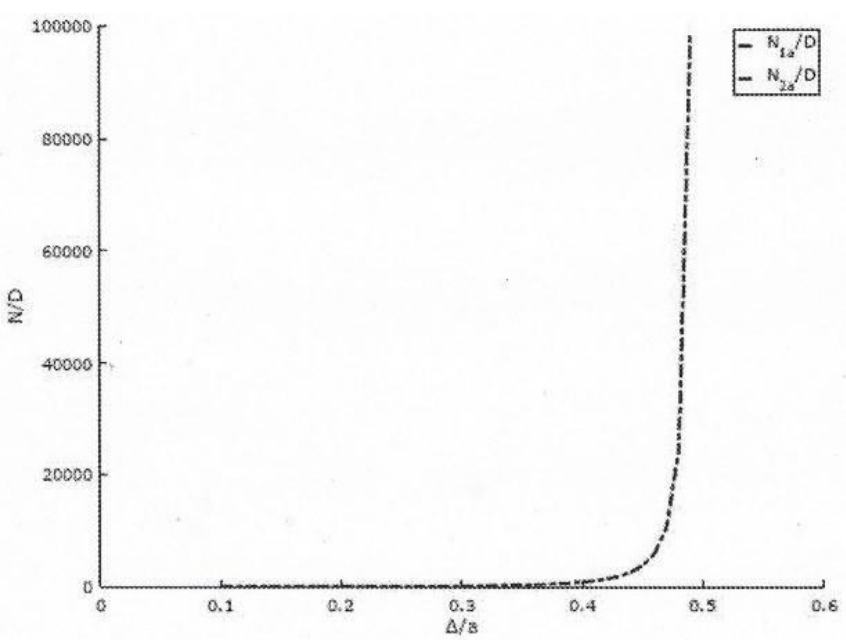

(c)

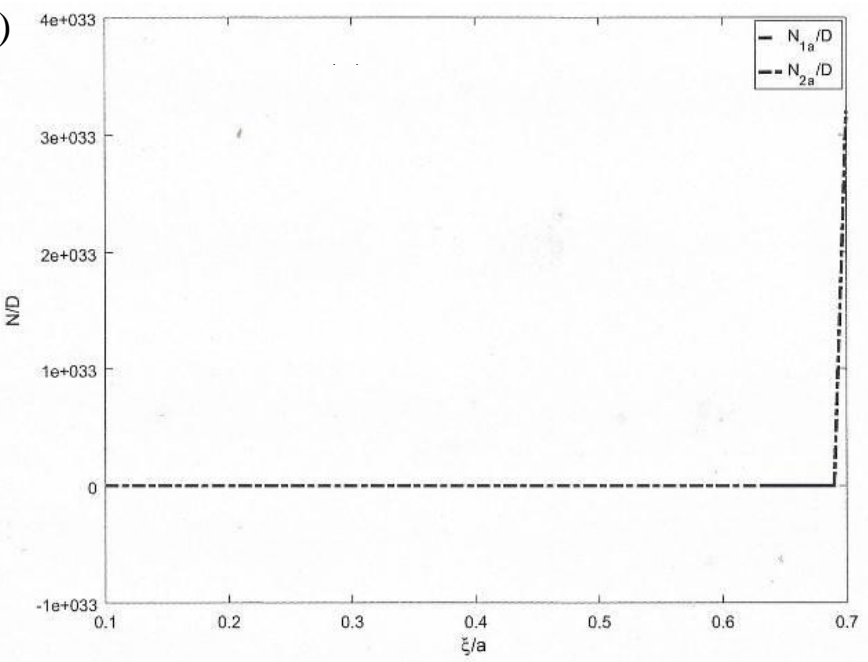

Fig. 4: Stability criteria for simply supported plates under intermediate load $N_{2}$ and end load $N_{1}$ vs. (a) different geometrical ratio $(a / b)$ values (b) different delta $(\Delta)$ width length values (c) different $\xi$ distance values. 
(a)

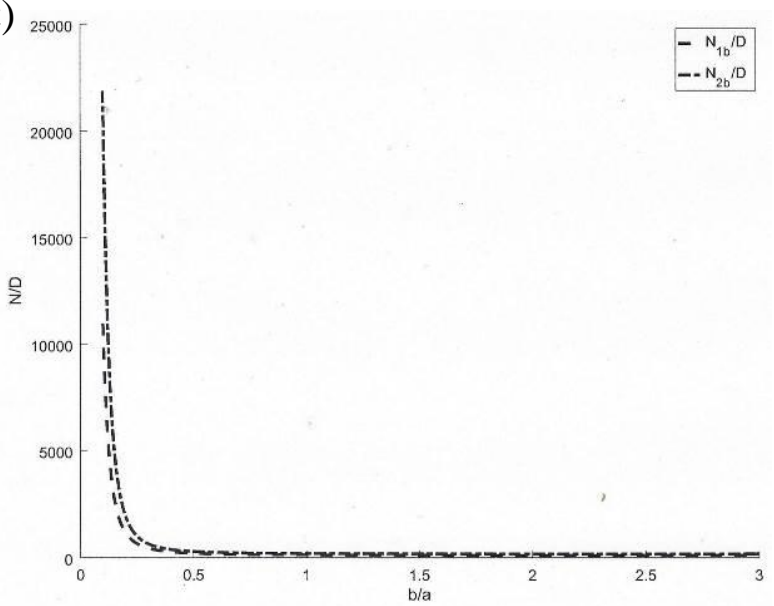

(b)

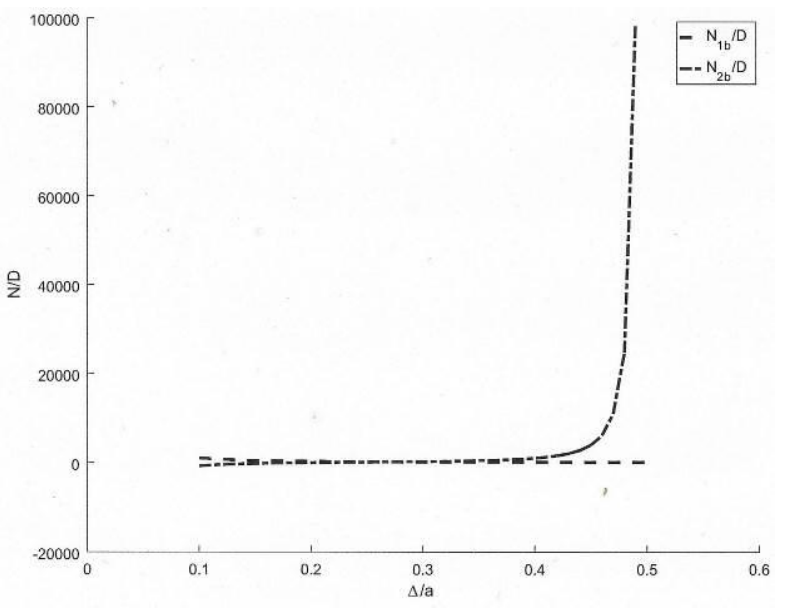

(c)

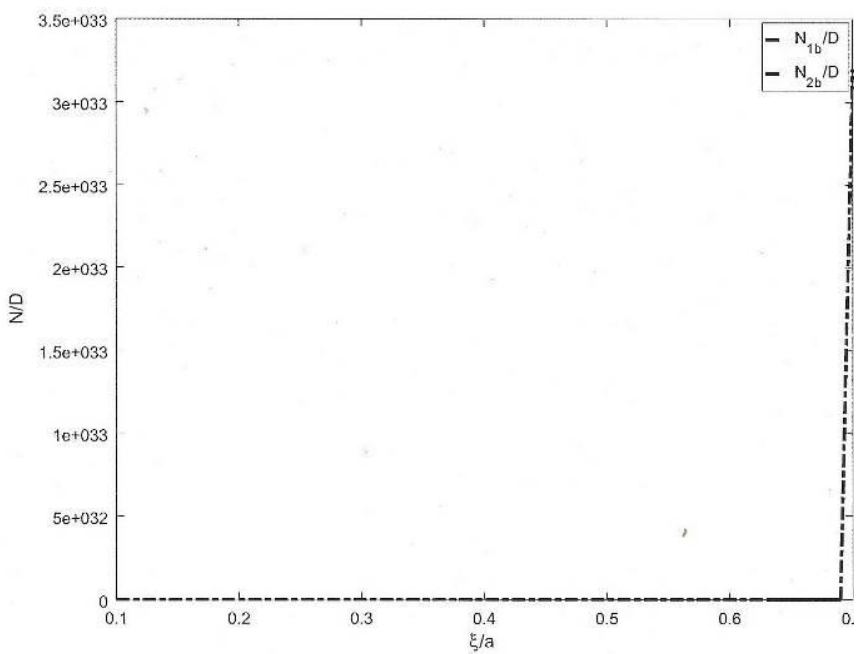

Fig. 5: Stability criteria for simply supported plates under intermediate load $N_{2}$ and end load $N_{1}$ vs. (a) different geometrical ratio $(a / b)$ values (b) different delta $(\Delta)$ width length values (c) different $\xi$ distance values. (a)

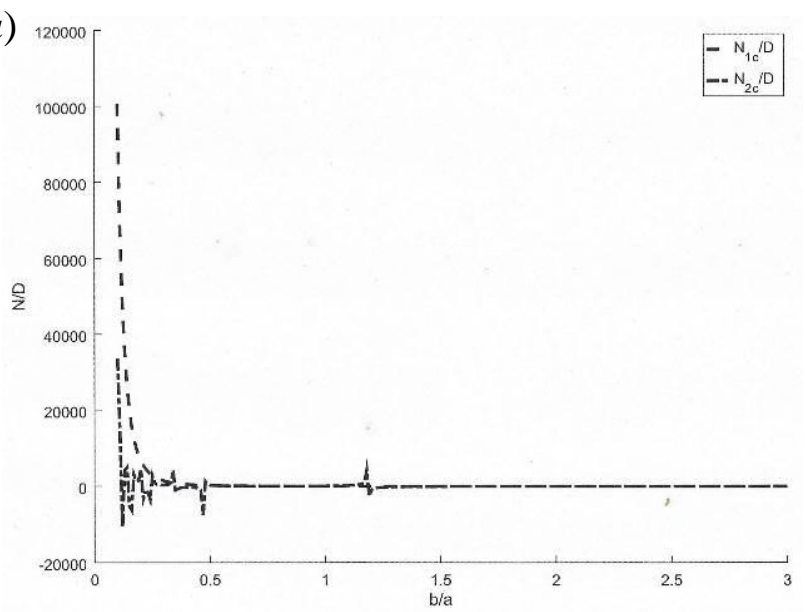

(b)
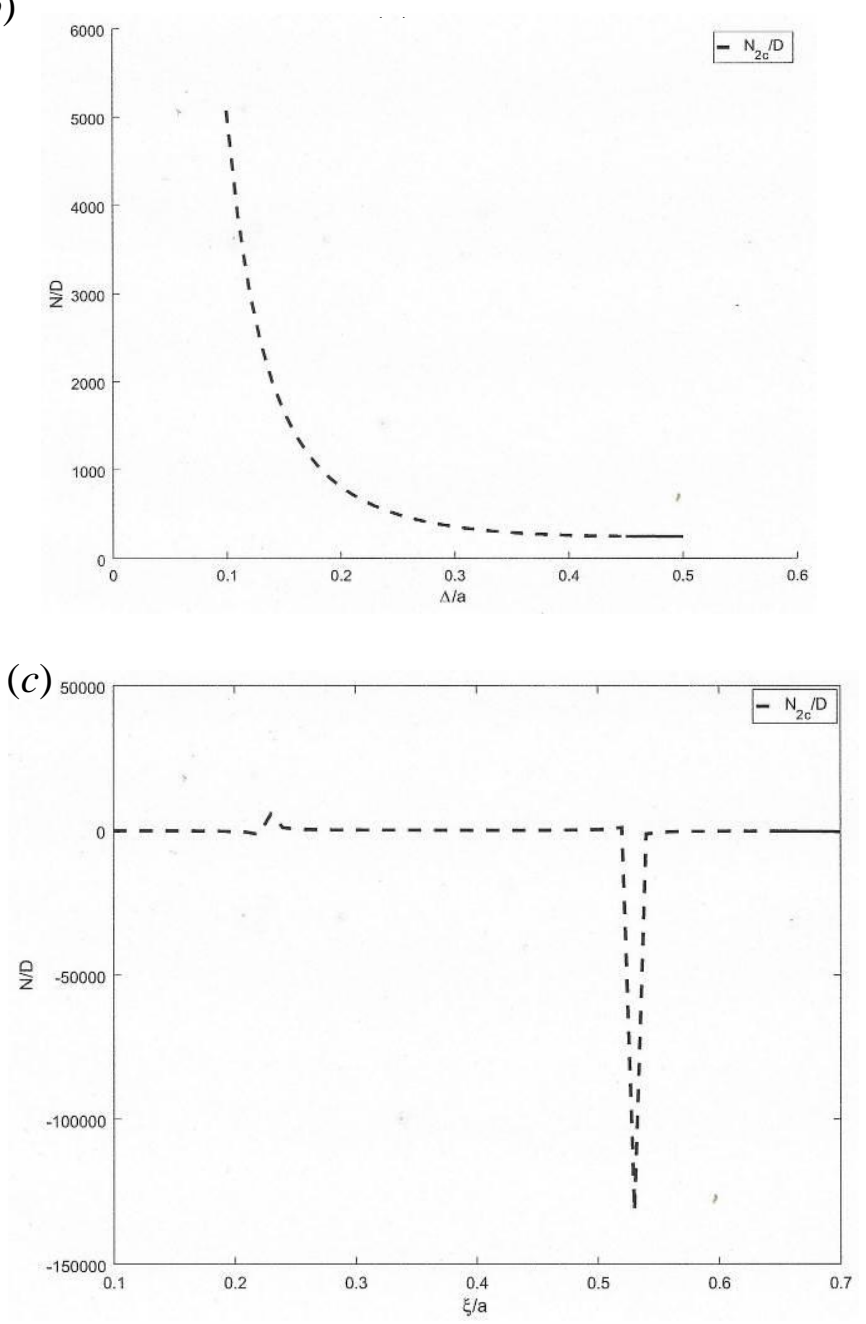

Fig. 6: Stability criteria for simply supported plates under intermediate load $N_{2}$ and end load $N_{1}$ vs. (a) different geometrical ratio $(a / b)$ values (b) different delta $(\Delta)$ width length values (c) different $\xi$ distance values. 
(a)

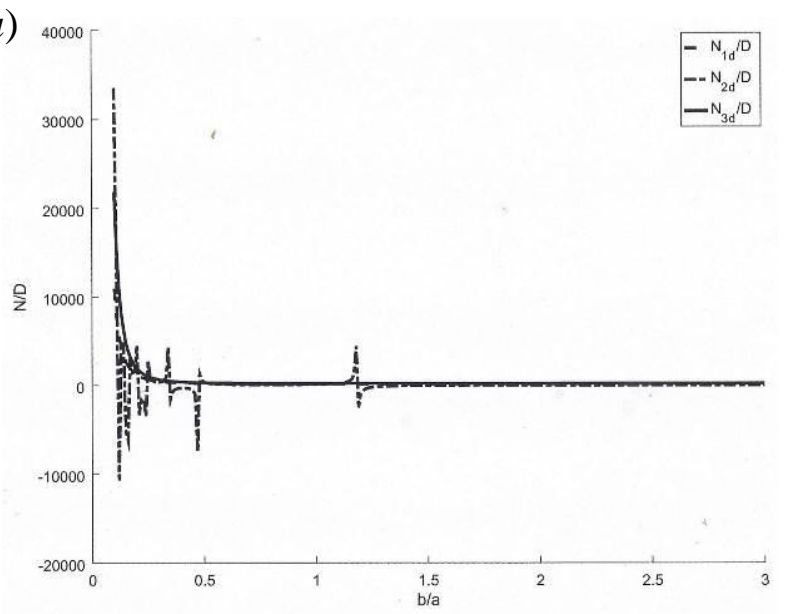

(b)

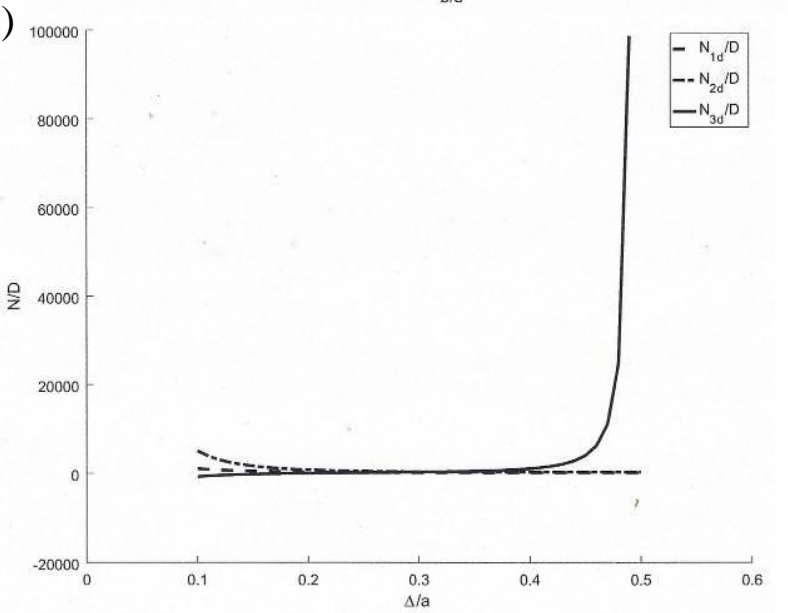

(c)

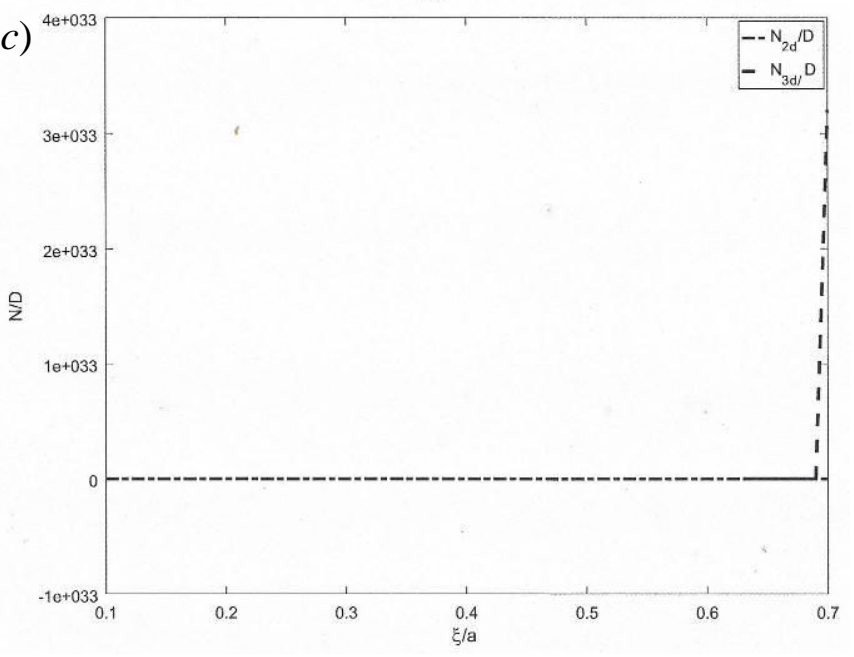

Fig. 7: Stability criteria for simply supported plates under intermediate load $N 2$ and end load $N 1$ vs. (a) different geometrical ratio $(a / b)$ values (b) different delta $(\Delta)$ width length values (c) different $\xi$ distance values.

\section{Conclusion}

Current study presents generalized static analytic solution for the critical buckling load of the simply supported rectangular plate subjected to the intermediate strip in-plane loading, based on the strain energy method. The expression was found to be dependent only on the strip thickness, strip location, plate width and stress magnitude. The developed solutions were found to agree quantitatively and qualitatively with the classical solution.

Compatible with the classical columns Euler method it was found that the plate stability is decreased with the increasing of the plate width. Accordingly, it can be assumed approximately that the minimum buckling load is linearly proportional to the geometrical ratio between plate length and plate width $(a / b)$.

Moreover, the strip width $(\Delta)$ parameter analysis has shown that increase is likely to cause buckling stress development due to the fact that the increase of the strip width is accompanied with larger induced stresses that accelerating the buckling process. In addition, strip location $(\xi)$ was found to be of importance due to the fact that the stresses acting in a further distance relative to the supports locations might accelerating the buckling process which is compatible with the Euler compressed column theory.

Additionally, expressions that includes both axial and transverse loads for different extended cases configurations were also derived and examined based on the strain energy method alongside explanation for possible applications (thin aluminum plate welding). In general view, it was found that the cases of combined axial and perpendicular loading action are less stabilized than cases where only one kind of loading configuration is participated.

Finally, comparison to literature references shows good qualitative agreement. Further studies in the field might include the dynamic case (with simulation performance) of plates and shells. Also, combination of out of plane load might contribute for the whole comprehension.

\section{References:}

[1] Brayan G. H., (1890) On the stability of a plane plata under thrusts in its own plane, with Applications to the "Buckling" of the Sides of a Ship, Camb. Phil. Proc., vi., 54-67, $28 \mathrm{C}$.

[2] Jang B. S., (2012) Topics in Ship Structural Design: Lecture 5 Buckling and ultimate strength of plates, OPen INteractive Structural Lab.

[3] Kumar A., Arakerimath R. (2015), A review 
and buckling analysis of stiffened plate, IOSR-JMCE, 01-11.

[4] [Helbig, D., Silva, C. C. C., Real, M. V., Santos, E. D., Isoldi, L. A., Rocha, L. A. O., (2016) Study about buckling phenomenon in perforated thin steel plates employing computational modeling and constructal design method. Latin American J. of Solids and Struct., 13, 10, 1912-1936.

[5] Pattnaik S. P., (2006) Prediction of bending waves in thin plates formed by buckling during rolling process, MM - thesis, B. Tech, Indian Institute of Technology, Kharagpur, India.

[6] Tran D. C., Tardif N., Limam A., (2015) Experimental and numerical modeling of flatness defects in strip cold rolling, Int. J. of Solids and Struct., 69-70, 343-349.

[7] Ari-Gur J., Simonetta, S. R., (1997) Dynamic pulse buckling of rectangular composite plates, Composites Part B 28B, 301 -308.

[8] Panda S. K., Ramachandra L. S., (2011) Buckling and Postbuckling Behavior of Cross-Ply Composite Plate Subjected to Nonuniform In-Plane Loads, Journal of Engineering Mechanics, 137, 9.

[9] Parry G., Cimetière A., Coupeau C., Colin J., Grilhé J., (2006) Stability diagram of unilateral buckling patterns of stripdelaminated films, Phys. Rev. E., 74, 066601.

[10] Najafizadeh M. N., Mahdavian M., Khazaeinejad P., (2010) Superposition buckling analysis of rectangular plates composed of functionally graded materials subjected to non- uniform distributed in-plane loading, Proc. of the Institution of Mechanical Engineers, Part C: Journal of Mechanical Engineering Science, 224, 2299-2307.

[11] Fazzolari F. A., (2016) Stability analysis of FGM sandwich plates by using variablekinematics Ritz models, Mechanics of Advanced Materials and Structures, 23, 9, 1104-1113.

[12] Majorana C. E., and Pomaro B., (2016) Dynamic Stability of Elastic Rectangular Plates with Viscoelasto-Damaged Constraints, Journal of Engineering Mechanics, ASCE, 142, 10, 04016077.

[13] Amoushahi H., (2018) Time depended deformation and buckling of viscoelastic thick plates by a fully discretized finite strip method using Third order shear deformation theory, European Journal of Mechanics / A Solids, 68, $38-52$.

[14] Ventsel E., Krauthammer T., (2011) Thin
Plates and Shells, Marcel Dekker, Inc., New York, Basel.

[15] Timoshenko S., Woinowsky-Krieger S., (1987) Theory of Plates and Shells, $2^{\text {nd }}$ ed. McGraw-Hill.

[16] Bulson P. S., (1970) The Stability of Flat Plates, Chatto \& Windus, London.

[17] Birman V., (2010) Plates and Shells, Encyclopedia of Aerospace Engineering (Eds. R. Blockley, W. Shvyy), J. Wiley, Struct. Mech. And Tech.

[18] Jaeger L.G., (1964) Elementary Theory of Elastic Plates: Ch. 4 - Membrane forces in the plate: stability, Pergamon Press, Oxford.

[19] Kubiak T., (2012) Nonlinear plate theory for postbuckling behaviour of thin-walled structures under static and dynamic load. Ch. 9 in: Nonlinearity, Bifurcation and Chaos Theory and Applications (Eds. J. Awrejcewics, P. Hagedorn), InTech, Rijeka.

[20] Maarefdoust M., Kadkhodayan M., (2015) Elastic/plastic buckling analysis of skew plates under in-plane shear loading with incremental and deformation theories of plasticity by GDQ method, J. Braz. Soc. Mech. Sci. Eng. 37, 761-776.

[21] Dima I., (2015) Buckling of Flat Thin Plates under Combined Loading, INCAS Bull., 7, 1, $83-96$.

[22] Poodeh F., Farhatnia F., Raeesi M., (2018) Buckling analysis of orthotropic thin rectangular plates subjected to nonlinear inplane distributed loads using generalized differential quadrature method, Int. J. Comp. Met. Eng. Sci. and Mech., 19, 2, 102-116.

[23] Rammerstorfer F. G., (2018) Buckling of elastic structures under tensile loads, Acta Mech., 229, 881-900.

[24] Yshii L. N., Lucena Neto E., Monteiro F. A. C., Santana R. C., (2018) Accuracy of the Buckling Predictions of Anisotropic Plates, $J$. Eng. Mech., 144, 8, 04018061.

[25] Xiang Y., Wang C. M., Kitipornchai S., (2003) Exact Buckling Solutions For Rectangular Plates Under Intermediate and End Uniaxial Loads, J. Eng. Mech., 129, 7.

[26] Yao L. K., He B., Zhang Y., Zhou W., (2015) Semi-Analytical Finite Strip Transfer Matrix Method for Buckling Analysis of Rectangular Thin Plates, Math. Problems in Eng., Article ID: 485686, 1-11.

[27] Mijušković O. Ćorić B., Šćepanović B., (2015) Accurate buckling loads of plates with different boundary conditions under arbitrary edge compression, Int. J. Mech. Sci., 101-102, 
309-323.

[28] Wang C. M., Chen Y., Xiang Y., (2004) Plastic buckling of rectangular plates subjected to intermediate and end inplane loads, Int. J. of Solids and Struct., 41, 42794297.

[29] Wang C. M., Chen Y., Xiang Y., (2004) Stability criteria for rectangular plates subjected to intermediate and end inplane loads, Thin-Walled Structures, 42, 119-136.

[30] Hwang I., Lee J. S., (2006) Buckling of Orthotropic Plates under Various Inplane Loads, KSCE Journal of Civil Engineering, 10, 5 .

[31] Stoll, F., (1994) Analysis of the snap phenomenon in buckled plates, Int. J. NonLinear Mechanics. 29, 2. 123-138.

[32] Shahwan, K. W., Waas, A. M., (1998) Buckling of Unilaterally Constrained Infinite Plates, JEM, 124, 2, 127-136.

[33] Reddy, J. N., (2007) Theory and analysis of elastic plates and shells, CRC Press, Taylor and Francis.

[34] Brunelle, E. J., and Robertson, S. R., (1974) Initially Stressed Mindlin Plates, 12, 8, AIAA J., 1036-1045.

[35] Xiang, Y., Wang C. M., Liew, K. M., Kitipornchai, S., (1993) Mindlin plate buckling with prebuckling in-plane deformation, J. Eng. Mech. 119, 1-18.

[36] Zhong, H., Gu, C., (2006) Buckling of Simply Supported Rectangular Reissner-Mindlin Plates Subjected to Linearly Varying In-Plane Loading, Tech. Notes, JEM, 132, 578-581.

[37] Bui, T. Q., Nguyen, M. N., Zhang, Ch., (2011) Buckling analysis of Reissner-Mindlin plates subjected to in-plane edge loads using a shear-locking-free and meshfree method, Eng. Analysis with Boundary Elem., 35, 10381053.

[38] Huang, J., Nguyen-Thanh, N., \& Zhou, K., (2017) Extended isogeometric analysis based on Bézier extraction for the buckling analysis of Mindlin-Reissner plates. Acta Mech., 228, 3077-3093.

[39] Reissner E., and Stein, M., (1951) Torsion and transverse bending of cantilever beam, NACA Tech. Note, Langley Aeronautical Laboratory Lamgley Field, 2369, 1-39.

[40] Berger, M. S., (1967) On von kármán's equations and the buckling of a thin elastic plate, I the clamped plate, Comm. On Pure \& App. Math., 687-719.

[41] Berger, M. S., (1968) On von Kármán's equations and the buckling of a thin elastic plate, II the clamped plate, Comm. On Pure \& App. Math., 227-241.

[42] Ueda, Y., Yao, T., (1985) The influence of complex initial deflection modes on the behaviour and ultimate strength of rectangular plates in compression, 5, 4 265-302.

[43] Belgacem H. V., Conti S., DeSimone, A., Muller, S., (2000) Rigorous Bounds for the Foppl-von Karman Theory of Isotropically Compressed Plates, J. Nonlinear Sci., 10, 661-683.

[44] Ibearugbulem M. O., Ezeh J. C., Ettu L. O., Oguaghamba O. A., (2015) Buckling and Postbuckling Strength of CSCS Thin Rectangular Plate, IJIRD, 4, 11, 206-212.

\section{Data Availability}

All data, models, and code generated or used during the study appear in the submitted article.

\section{No Interest Conflict}

The corresponding author (Jacob Nagler) states that there is no conflict of interest.

\section{Sources of Funding for Research Presented in a Scientific Article or Scientific Article Itself \\ No funding is involved.}

\section{Creative Commons Attribution License 4.0 (Attribution 4.0 International, CC BY 4.0)}

This article is published under the terms of the Creative Commons Attribution License 4.0 https://creativecommons.org/licenses/by/4.0/deed.en US 